\title{
Two-Stage Dynamic Pricing and Advertising Strategies for Online Video Services
}

\author{
Zhi Li ${ }^{1,2}$ and De-qing $\operatorname{Tan}^{3}$ \\ ${ }^{1}$ School of Management, Tianjin Polytechnic University, Tianjin 300387, China \\ ${ }^{2}$ College of Management and Economics, Tianjin University, Tianjin 300072, China \\ ${ }^{3}$ School of Economics \& Management, Southwest Jiaotong University, Chengdu 610031, China \\ Correspondence should be addressed to Zhi Li; lizhi@tjpu.edu.cn
}

Received 4 May 2017; Revised 26 August 2017; Accepted 29 August 2017; Published 18 October 2017

Academic Editor: Cemıl Tunç

Copyright (C) 2017 Zhi Li and De-qing Tan. This is an open access article distributed under the Creative Commons Attribution License, which permits unrestricted use, distribution, and reproduction in any medium, provided the original work is properly cited.

\begin{abstract}
As the demands for online video services increase intensively, the selection of business models has drawn the great attention of online providers. Among them, pay-per-view mode and advertising mode are two important resource modes, where the reasonable fee charge and suitable volume of ads need to be determined. This paper establishes an analytical framework studying the optimal dynamic pricing and advertising strategies for online providers; it shows how the strategies are influenced by the videos available time and the viewers' emotional factor. We create the two-stage strategy of revenue models involving a single fee mode and a mixed fee-free mode and find out the optimal fee charge and advertising level of online video services. According to the results, the optimal video price and ads volume dynamically vary over time. The viewer's aversion level to advertising has direct effects on both the volume of ads and the number of viewers who have selected low-quality content. The optimal volume of ads decreases with the increase of ads-aversion coefficient, while increasing as the quality of videos increases. The results also indicate that, in the long run, a pure fee mode or free mode is the optimal strategy for online providers.
\end{abstract}

\section{Introduction}

Apart from text and images, video is the most important form of online media, which has been widely regarded as the future of media on the Internet. According to Cisco Visual Networking Index $2015-2020$ prediction, by 2020 , $82 \%$ of viewer Internet traffic will be video and total global Internet traffic will increase at $22 \%$ per year. High-quality online video service brings not only profits but also reputation and user reliability to the companies. The latter is rather more important for company's long term life cycle. For example, Youtube has attracted over a billion users all over world, because it offers free video services, selected advertising options, and a much faster streaming ability thanks to its great technologies such as Content Delivery Network (CDN) and Google File System (GFS). In other words, Youtube has paid a lot of attention to user experience and thus gains a large and stable user group, which makes Youtube one of the most famous video websites in the world. However, in 2014 Youtube annual revenue was posted at about $\$ 4$ billion, which accounted for about $6 \%$ of Google's overall sales, which increased to $\$ 9$ billion in 2015. This indicates that there will be a large potentiality for Youtube to be more profitable. Not only does Youtube exist, but also there are various Internet video sites such as Vimeo (US), Hulu (US), Youku (China), Dailymotion (France), Niconico (Japan), and Pandora TV (South Korea), competing in the online video market all over the world.

As the online video service has a glorious future, it is attractive and important to investigate its business models. There are several profiting modes, which mainly include three patterns: sales or redistribution of copyrights for certain films, charges for video services, and advertising revenue. In this paper, we will discuss the two latter cases dealing with online video users. Many video websites adopt the strategy of providing free video services to maximize membership. The revenues of this mode mainly depend on advertising. However, recent studies report that, with only advertising revenue, it is not sufficient for Internet-based 
companies [1, 2]. According to Weiss [3], fee charge mode is necessary and becoming more popular in web content market. Hence, the strategy of combining fee charge and advertising has emerged, which has been regarded as the mainstream business model, such as Youku, the most famous video service website in China, which offers free videos with ads as well as a paid membership with a higher priority and no ads. Although the mixed strategy is popular, it also raises the problem of how to balance the revenue from fee charging and advertising. For Internet video services, higher prices result in fewer viewers, and a large amount of ads will also scare them away. In this sense, there exists a trade-off between video service price and the amount of ads.

In order to deal with this trade-off, some video websites adopt strategy that allows viewers to preview samples before buying. It might be feasible, because online video is a sort of information goods as well as experience goods, which could be valued according to viewers' perceived experience [4]. Hence, free samples are introduced into the business models with the purpose of ensuring that viewers have an actual experience with information goods $[5,6]$. This humanized experience mode is helpful to set up reasonable price and ads volume for information goods. With significant increasing number of the Internet users, the pricing and advertising models have attracted attention of many researchers. Riggins [7] develops a separating equilibrium model for fee-based and ads sponsored-based websites and examines a monopolist's choice of web content price and quality. In another study, Prasad et al. [8] suggest different optimal strategies for content firms depending on the context: pure pay-per-view strategy for high income users, who are willing to pay the advertising avoidance fee, and free strategy based on advertisement for high advertising sales with low-quality content. The study derives the optimal condition for each strategy. Halbheer et al. [9] investigates digital content strategies: the sampling, paid content, and free content strategies for online publishers. Results show that the optimal strategy is determined by the relationship between advertising effectiveness and content quality. Chellappa and Shivendu [1] examine pricing and sampling strategies for digital experience goods; through a two-stage viewer piracy behavior model they obtain the optimal price and sample size. Peitz and Valletti [10] introduce the factor of viewer's favorite type into paytv and free-to-air models in the two-side market. The results indicate that the more sensitive the viewers are to ads the more the differentiated content should be. Kwon [11] constructs a stochastic model for online display advertising contracts and explores how pricing and contract components affect the optimal display strategies. Lin et al. [12] assert that a mixed strategy of paid and free content is most favorable for content firms in a monopoly position, and in a duopoly state, only one firm is able to employ the mixed strategy.

The papers mentioned above apply static models and optimize the maximum revenue using simple first-order and second-order conditions without considering the effect of time on the optimal strategy. However, a few papers do take into consideration the time periods in different ways and provide a dynamic analysis to study the optimal content price and the volume of ads in websites. For instance,
Dewan et al. [13] develop an advertising revenue model and obtain the optimal dynamic ads volume for the manager of Internet. Kumar and Sethi [2] study dynamic pricing and advertising for web content providers and test the influence of parameters such as horizon length, cost of serving, natural growth rate, and content's utility factor on the optimal strategies. Based on the pricing strategy, $\mathrm{Na}$ et al. [14] categorize Korea's digital content firms' strategies into three groups: pure pay-per-view strategy, free strategy based on advertisement, and a mixed strategy; then they apply the metafrontier analysis method to estimate each group's efficiency value. As extension, our model differs from theirs by further considering the hybrid business models with the type of viewers and emotional factor varying over time. In this paper, we will introduce two business models including the fee mode and the free mode that allow viewers to select. There are two cases under consideration, the single fee mode case and the mixed fee-free mode case. In the mixed fee-free mode, viewers could maximize their utility by selecting within the two modes. Based on the emotional factor, the type of viewers, and the perceived value, we propose a model for finding the optimal dynamic fee charge and ads level over a finite time interval, to maximize the total profit of online video providers. To the best of our knowledge, it is the first analysis with a continuous time model and emotional factor in this setting. The paper is innovative and new, which firstly applies Hamilton maximum principle to solve the optimal pricing and advertising strategies for online video services.

\section{The Model}

Consider that the online provider offers two types of Internet video contents: high-quality content without ads requiring a fee to access and low-quality content with ads but free of charge. There will be previews for viewers to have a general judge over the video service. Assume that for high-quality and low-quality video services, the viewer's average perceived values are $v_{h}$ and $v_{l}$, respectively, where $v_{h}>v_{l}>0$ [15]. Since different viewers react differently to the same video content, they can be classified further by their types. For this model, we define high-quality type preferred viewers as those who are more sensitive to video quality; thus they have more willingness to choose the fee mode. Similarly, defined economy preferred viewers are those who are unwilling to pay for online videos but may tolerate advertising; thus they prefer to choose the free mode. Meanwhile we assume that (as well as in [16]) viewer's type $\theta$ follows a uniform distribution on $[0,1]$, which is a factor indicating viewers' willingness to pay for service. When $\theta$ is close to 1 , the viewers are more likely to choose the fee mode, and vice versa.

In this paper, the price of online video services and the amount of ads are functions of time $t$ denoted by $p_{t}$ and $q_{t}$, respectively. Based on above assumptions, define the viewer expected utility such that a viewer of type $\theta_{h}$ receiving from high-quality content is $U_{h}(t)=\theta_{h} v_{h}-p_{t}$ and from low-quality content is $U_{l}(t)=\theta_{l} v_{l}-\beta q_{t}$. In the free mode, viewers have to view ads that come with video content. Thus, the advertising is regarded as a nuisance that could reduce viewers' utility $[17,18]$. Based on this fact, considering viewers' emotional 
factor that could affect the viewing mode, we define $\beta \in(0,1)$ as a constant representing ads-aversion coefficient. When $\beta$ value gets closer to 1 , it means viewers are less tolerant to ads. We assume that the marginal cost of producing a product or introducing an advertisement is zero. Viewers have to view ads that come in order to access the free product. To mirror the properties of pricing and advertising strategies, let the cost of setting ads be zero [7], and the producing cost of content is negligible on Internet.

In the following part, we will introduce the single fee mode and the mixed fee-free mode, where the optimal video price and ads volume are resolved to obtain the optimal business strategies for online video services.

2.1. Case I: Fee Mode in Time Interval $\left(0, t_{1}\right)$. At time $t$, when $U_{h}(t)=\theta_{h} v_{h}-p_{t} \geq 0, \theta_{h} \geq p_{t} / v_{h}$, viewers choose the fee mode. Define the total volume of viewers as 1 . Let $x_{h, t}$ be the number of viewers who have selected high-quality content. The current density of viewers $\dot{x}_{h, t}=d x_{h, t} / d t$ is expressed as

$$
\begin{aligned}
& D_{h, t}=\int_{p_{t} / v_{h}}^{\theta_{h}} d \theta=\theta_{h}-\frac{p_{t}}{v_{h}}=1-x_{h, t}-\frac{p_{t}}{v_{h}}, \\
& \dot{x}_{h, t}=1-x_{h, t}-\frac{p_{t}}{v_{h}} .
\end{aligned}
$$

Let $\dot{x}_{h, t}=d x_{h, t} / d t$ be the change rate of the number of high type viewers and define $\dot{x}_{h, t}=D_{h, t}$. We denote that online providers make profits over the planning horizon with length $t_{1}$, and then the profit is given as

$$
\Pi_{\mathrm{I}}=\int_{0}^{t_{1}} p_{t} \dot{x}_{h, t} d t
$$

In case $\mathrm{I}$, it needs to find out the optimal price $p_{t}^{*}$ which maximizes the profit $\Pi_{\mathrm{I}}$. The objective function for the provider is

$$
J_{1}=\max \int_{0}^{t_{1}} p_{t} \dot{x}_{h, t} d t
$$

This is a dynamic optimization problem, which can be solved by the modern control theory Hamiltonian Method. In the model, a dot above a variable is defined as the first derivative with respect to time. We formulate Hamiltonian function as follows:

$$
H_{1}=p_{t}\left(1-x_{h, t}-\frac{p_{t}}{v_{h}}\right)+\lambda_{0, t}\left(1-x_{h, t}-\frac{p_{t}}{v_{h}}\right),
$$

where $p_{t}$ is a control variable and $\lambda_{0, t}$ is a time-varying constructor variable. According to Hamiltonian principle [19, 20], the necessary conditions for the optimal control are listed as follows:

$$
\begin{aligned}
\frac{\partial H_{1}}{\partial p_{t}} & =0 \\
\dot{\lambda}_{0, t} & =-\frac{\partial H_{1}}{\partial x_{h, t}} .
\end{aligned}
$$

Since $p_{t}$ is unconstrained, the optimal price should satisfy the first-order condition $\partial H_{1} / \partial p_{t}=0$, from which we obtain

$$
p_{t}=\frac{v_{h}\left(1-x_{h, t}\right)-\lambda_{0, t}}{2} .
$$

From (1) and (6), the increment rate of viewers who select high-quality content $\dot{x}_{h, t}$ is given by

$$
\dot{x}_{h, t}=\frac{\lambda_{0, t}+v_{h}\left(1-x_{h, t}\right)}{2 v_{h}} .
$$

Since Lagrangian multiplier satisfies the condition $\dot{\lambda}_{0, t}=$ $-\partial H_{1} / \partial x_{h, t}$, we have the following equation:

$$
\dot{\lambda}_{0, t}=\frac{\lambda_{0, t}+v_{h}\left(1-x_{h, t}\right)}{2} .
$$

Deduced from (7) and (8), we obtain

$$
\dot{\lambda}_{0, t}=v_{h} \dot{x}_{h, t} .
$$

Equation (9) is a first-order differential equation. With border conditions $x_{h, t=0}=0$ and $\lambda_{0}\left(t_{0}\right)=0$, final solutions are achieved as $\lambda_{0, t}=v_{h}\left(t-t_{1}\right) /\left(2+t_{1}\right), x_{h, t}=t /\left(2+t_{1}\right)$.

We substitute the values of $\lambda_{0, t}$ and $x_{h, t}$ into (6) and derive the optimal price of online video services $p_{t}^{*}=v_{h}\left(1+t_{1}\right.$ $t) /\left(2+t_{1}\right)$ that could make the maximum profit in fee mode with time interval $\left(0, t_{1}\right)$.

2.2. Case II: Mixed Fee-Free Mode in Time Interval $\left(0, t_{1}\right)$. In this case, there exists both fee and free modes in online video service market. Viewers could choose any mode as their willingness. Since the newly arriving video contents are more attractive, viewers prefer to pay for the new ones. With this consideration, introduce a critical time point $t_{0}$, which divides the whole business time into two stages: in time interval $\left(0, t_{0}\right)$, providers employ the fee mode; in time interval $\left(t_{0}, t_{1}\right)$, providers employ the mixed fee-free mode.

Stage 1 (fee mode $\left.\left(0 \leq t \leq t_{0}\right)\right)$. Here we make an assumption that the optimal solution for fee model is the same as case I. This assumption stands when $t_{1}$ is not infinite and for the purpose of simplifying the model. It means that in time interval $0 \leq t \leq t_{0}$, the optimal price of online video services is $p_{t}^{*}=v_{h}\left(1+t_{1}-t\right) /\left(2+t_{1}\right)$.

Stage 2 (fee-free mode $\left(t_{0}<t \leq t_{1}\right)$ ). At time $t_{0}$, introduce the free mode into the single fee mode in online video market, and therefore there exists both fee and free modes at the same time. The advertising in free mode will attenuate viewers' selection. In the fee mode, we adopt a scaled price of Stage 1 for Stage 2 for simplicity. Denote $\gamma$ as a regular factor in $(0,1]$, which determines the price in Stage 2 as $\gamma p_{t}$. For viewers, if $U_{h}(t)>U_{l}(t)$ fee mode is preferred, otherwise, free mode is better. Furthermore, we have the following results:

If $0 \leq \theta v_{l}-\beta q_{t}<\theta v_{h}-\gamma p_{t}, \theta>\left(\gamma p_{t}-\beta q_{t}\right) /\left(v_{h}-v_{l}\right) \geq 0$, viewers prefer the fee mode.

If $0 \leq \theta v_{h}-\gamma p_{t} \leq \theta v_{l}-\beta q_{t}, \beta q_{t} / v_{l}<\theta<\left(\gamma p_{t}-\right.$ $\left.\beta q_{t}\right) /\left(v_{h}-v_{l}\right)$, viewers prefer the free mode. 
It can be found that $\tilde{\theta}=\left(\gamma p_{t}-\beta q_{t}\right) /\left(v_{h}-v_{l}\right)$ is the critical point of viewer type.

Denote $x_{l, t}$ as the number of viewers who have viewed low-quality content; thus the current densities of different viewers are defined as

$$
\begin{aligned}
& D_{h, t}=\theta_{h}-\frac{\gamma p_{t}-\beta q_{t}}{v_{h}-v_{l}}, \\
& D_{l, t}=\frac{\gamma p_{t}-\beta q_{t}}{v_{h}-v_{l}}-\frac{\beta q_{t}}{v_{l}} .
\end{aligned}
$$

Since $\dot{x}_{h, t}=D_{h, t}, \dot{x}_{l, t}=D_{l, t}$, we have

$$
\begin{aligned}
& \dot{x}_{h, t}=1-\frac{t_{0}}{2+t_{0}}-\frac{\gamma p_{t}-\beta q_{t}}{v_{h}-v_{l}}-x_{h, t}-x_{l, t}, \\
& \dot{x}_{l, t}=\frac{\gamma p_{t}-\beta q_{t}}{v_{h}-v_{l}}-\frac{\beta q_{t}}{v_{l}},
\end{aligned}
$$

where $\dot{x}_{h, t}$ is the density of arriving viewers who select fee mode and $\dot{x}_{l, t}$ is the density of viewers who select free mode. The profit of fee-free mode is given as

$$
\Pi_{\mathrm{II}}=\int_{t_{0}}^{t_{1}}\left(\gamma p_{t} \dot{x}_{h, t}+k q_{t} \dot{x}_{l, t}\right) d t,
$$

where $k$ is the price of ads per unit. In Stage 2 , it needs to find the optimal ads volume $q_{t}^{*}$ to maximize profit $\Pi_{\text {II }}$. Over time period $t_{0}<t \leq t_{1}$, the objective function for the provider is

$$
J_{2}=\max \int_{t_{0}}^{t_{1}}\left(\gamma p_{t} \dot{x}_{h, t}+k q_{t} \dot{x}_{l, t}\right) d t,
$$

subject to (14) and (15):

$$
\begin{aligned}
& \dot{x}_{h, t}=1-\frac{t_{0}}{2+t_{0}}-\frac{\gamma p_{t}-\beta q_{t}}{v_{h}-v_{l}}-x_{h, t}-x_{l, t}, \\
& \dot{x}_{l, t}=\frac{\gamma p_{t}-\beta q_{t}}{v_{h}-v_{l}}-\frac{\beta q_{t}}{v_{l}} .
\end{aligned}
$$

The Hamiltonian function is given as follows:

$$
\begin{aligned}
H_{2}= & \left(\gamma p_{t}+\lambda_{1, t}\right)\left(\frac{2}{2+t_{0}}-\frac{\gamma p_{t}-\beta q_{t}}{v_{h}-v_{l}}-x_{h, t}-x_{l, t}\right) \\
& +\left(\lambda_{2, t}+k q_{t}\right)\left(\frac{\gamma p_{t}-\beta q_{t}}{v_{h}-v_{l}}-\frac{\beta q_{t}}{v_{l}}\right),
\end{aligned}
$$

where $q_{t}$ is the variable, and $p_{t}=v_{h}\left(1+t_{1}-t\right) /\left(2+t_{1}\right)$ is given in Stage 1. $\lambda_{1, t}$ and $\lambda_{2, t}$ are the two constructor variables corresponding to the state variables $x_{h, t}$ and $x_{l, t}$ [20]. Based on Hamiltonian principle, $q_{t}, \lambda_{1, t}, \lambda_{2, t}, x_{h, t}$, and $x_{l, t}$ satisfy the following equations:

$$
\begin{aligned}
& \frac{\partial H_{2}}{\partial q_{t}}=0 \\
& \dot{\lambda}_{1, t}=-\frac{\partial H_{2}}{\partial x_{h, t}}, \\
& \dot{\lambda}_{2, t}=-\frac{\partial H_{2}}{\partial x_{l, t}} .
\end{aligned}
$$

Since $q_{t}$ is unconstrained, $\partial H_{2} / \partial q_{t}=0$, the number of ads $q_{t}$ is obtained by

$$
q_{t}=\frac{\gamma}{2 \beta k\left(2+t_{1}\right)}\left((\beta+k) v_{l}+\left(t_{1}-t\right)\left(k v_{l}+\beta v_{h}\right)\right) .
$$

Since Lagrange's equation satisfies $\dot{\lambda}_{1, t}=-\partial H_{2} / \partial x_{h, t}, \dot{\lambda}_{2, t}=$ $-\partial H_{2} / \partial x_{l, t}$, we have the following equations:

$$
\begin{aligned}
& \dot{\lambda}_{1, t}=-\frac{\partial H_{2}}{\partial x_{h, t}}=\gamma p_{t}+\lambda_{1, t}, \\
& \dot{\lambda}_{2, t}=-\frac{\partial H_{2}}{\partial x_{l, t}}=\gamma p_{t}+\lambda_{1, t} .
\end{aligned}
$$

Through solving first-order differential equations (19) and with border condition $\lambda_{1, t}=\lambda_{2, t}=0$, we obtain

$$
\lambda_{1, t}=\lambda_{2, t}=-\frac{\gamma v_{h}\left(t_{1}-t\right)}{2+t_{1}} .
$$

From (14), (15), (18), (20), and $p_{t}$ we have the following results:

$$
\begin{aligned}
q_{t} & =\frac{\gamma}{2 \beta\left(2+t_{1}\right)}\left((\beta+k) v_{l}+\left(t_{1}-t\right)\left(\beta v_{h}+k v_{l}\right)\right), \\
x_{l, t} & =\frac{\gamma v_{h}}{2\left(v_{h}-v_{l}\right)\left(2+t_{1}\right)}\left[\left(1-\frac{\beta}{k}\right)\left(t-t_{0}\right)\right. \\
& \left.+\left(1-\frac{\beta v_{h}}{k v_{l}}\right)\left(t_{1}\left(t-t_{0}\right)-\frac{t^{2}-t_{0}^{2}}{2}\right)\right] .
\end{aligned}
$$

In addition, from the existing results, we can resolve $x_{h, t}$ considering (6), (14), (18), (22), and the border condition $x_{h, t}\left(t_{0}\right)=t_{0} /\left(2+t_{1}\right)$; the following formulation is given:

$$
\begin{aligned}
x_{h, t} & =\frac{\gamma v_{h}}{2\left(v_{h}-v_{l}\right)\left(2+t_{1}\right)}\left[\frac{1}{2}\left(1-\frac{\beta v_{h}}{k v_{l}}\right) t^{2}\right. \\
& +\left(\frac{\beta v_{h}}{k v_{l}}-\frac{v_{l}}{v_{h}}-t_{1}+\frac{\beta v_{h}}{k v_{l}} t_{1}\right) t-2+\left(\frac{\beta}{k}+2\right) \frac{v_{l}}{v_{h}} \\
& -\frac{\beta v_{h}}{k v_{l}}+\left(\frac{v_{l}}{v_{h}}+\frac{\beta}{k}-1-\frac{\beta v_{h}}{k v_{l}}\right) t_{1}+\left(1-\frac{\beta}{k}\right) t_{0} \\
& \left.+\left(1-\frac{\beta v_{h}}{k v_{l}}\right)\left(t_{0} t_{1}-\frac{t_{0}^{2}}{2}\right)\right]+C_{h} e^{-t}+\frac{2}{2+t_{0}},
\end{aligned}
$$

with

$$
\begin{aligned}
C_{h} & =e^{-t_{0}}\left(\frac{t_{0}}{2+t_{1}}-\frac{2}{2+t_{0}}\right. \\
& -\frac{\gamma v_{h}}{2\left(v_{h}-v_{l}\right)\left(2+t_{1}\right)}\left[\frac{1}{2}\left(1-\frac{\beta v_{h}}{k v_{l}}\right) t_{0}^{2}\right. \\
& +\left(\frac{\beta v_{h}}{k v_{l}}-\frac{v_{l}}{v_{h}}-t_{1}+\frac{\beta v_{h}}{k v_{l}} t_{1}\right) t_{0}-2+\left(\frac{\beta}{k}+2\right) \frac{v_{l}}{v_{h}} \\
& -\frac{\beta v_{h}}{k v_{l}}+\left(\frac{v_{l}}{v_{h}}+\frac{\beta}{k}-1-\frac{\beta v_{h}}{k v_{l}}\right) t_{1}+\left(1-\frac{\beta}{k}\right) t_{0} \\
& \left.\left.+\left(1-\frac{\beta v_{h}}{k v_{l}}\right)\left(t_{0} t_{1}-\frac{t_{0}^{2}}{2}\right)\right]\right) .
\end{aligned}
$$


Finally, we obtain the optimal trajectories for $x_{l, t}, \lambda_{1, t}$, and $\lambda_{2, t}$ in (20) and (22) and the optimal number of ads $q_{t}^{*}=$ $\left(\gamma / 2 \beta\left(2+t_{1}\right)\right)\left((\beta+k) v_{l}+\left(t_{1}-t\right)\left(\beta v_{h}+k v_{l}\right)\right)$ that makes the maximization of profit.

\section{The Optimal Strategy}

Based on the hybrid business models discussed in the above section, we can draw the following conclusions on optimal strategies, which are useful to gain insights into factors affecting the optimal price and quantity.

\subsection{The Optimal Pricing Strategy for Case I}

Proposition 1. In the single fee mode, it is optimal to set $p_{t}^{*}=$ $v_{h}\left(1+t_{1}-t\right) /\left(2+t_{1}\right), 0 \leq t \leq t_{1}$, in order to attend the maximum profit.

Proposition 1 shows that the optimal pricing strategy is determined by the relation between high-quality value and time.

Lemma 2. The optimal price $p_{t}^{*}$ is increasing as perceived value $v_{h}$ of high-quality video increases, but it is decreasing as time tincreases.

Proof. By taking the high-quality value and time partial derivatives of the optimal price, we obtain $\partial p_{t}^{*} / \partial v_{h}=v_{h}(1+$ $\left.t_{1}-t\right) /\left(2+t_{1}\right) \geq 0, \partial p_{t}^{*} / \partial t=-v_{h} /\left(2+t_{1}\right) \leq 0$, which supports Lemma 2.

Lemma 2 says, as higher perceived value obtained from high-quality content video, the higher fee charge could be realized.

\subsection{The Optimal Pricing-Advertising Strategy for Case II}

Proposition 3. In the mixed fee-free mode of online video market, it is optimal for online providers to adopt a paid content strategy as $p_{t}^{*}=\gamma v_{h}\left(1+t_{1}-t\right) /\left(2+t_{1}\right), 0 \leq t \leq t_{1}$; it is optimal to adopt a free content strategy as $q_{t}^{*}=\left(\gamma / 2 \beta\left(2+t_{1}\right)\right)\left((\beta+k) v_{l}+\right.$ $\left.\left(t_{1}-t\right)\left(k v_{l}+\beta v_{h}\right)\right), t_{0}<t \leq t_{1}$.

Proposition 3 shows the mixed fee-free strategy with the optimal price of online video services and the optimal number of ads. This proposition is validated in Section 2 discussion by means of Hamiltonian principle. As in the benchmark case, if viewers care more about the high-quality value of online video content, it is optimal for providers to employ the fee strategy; if the viewers are not willing to pay for the online content, meanwhile, they would rather accept ads (the value of ads-aversion coefficient is small); it is optimal for providers to employ the free strategy.

Lemma 4. The optimal number of ads $q_{t}^{*}$ is increasing as either high-quality value $v_{h}$ or low-quality value $v_{l}$ increases, while it is decreasing as either ads-aversion coefficient $\beta$ or time $t$ increases.

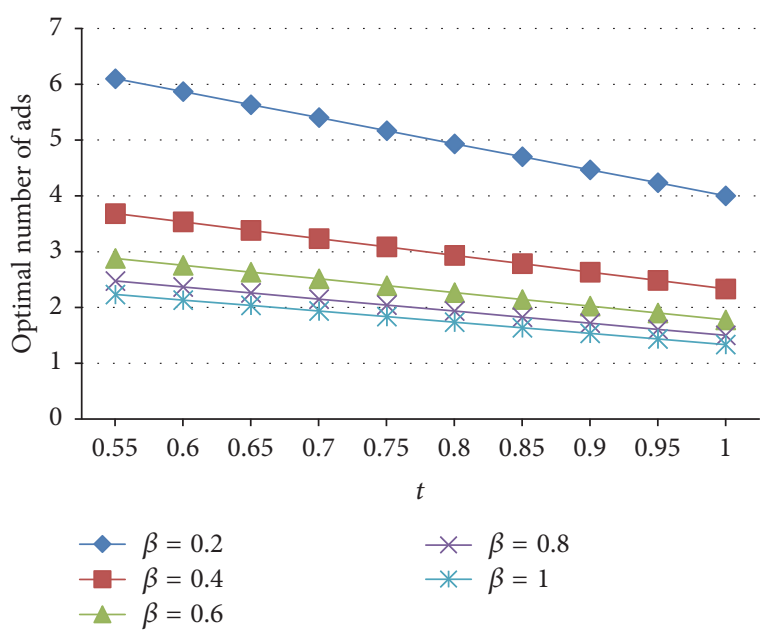

FIGURE 1: The optimal number of ads with respect to time $t$.

Proof. For the optimal number of ads $q_{t}^{*}$, we take the first derivative of $q_{t}^{*}$ versus $v_{h}, v_{l}$, and $t$ and then obtain $\partial q_{t}^{*} / \partial v_{h}=$ $\gamma v_{h}\left(t_{1}-t\right) / 2\left(2+t_{1}\right) \geq 0, \partial q_{t}^{*} / \partial v_{l}=\left(\gamma(\beta+k)+\left(t_{1}-t\right) k\right) / 2 \beta(2+$ $\left.t_{1}\right) \geq 0, \partial q_{t}^{*} / \partial t=-\gamma\left(\beta v_{h}+k v_{l}\right) / 2 \beta\left(2+t_{1}\right)<0$.

Through simple transform $q_{t}^{*}=\left(\gamma / 2 \beta\left(2+t_{1}\right)\right)\left((\beta+k) v_{l}+\right.$ $\left.\left(t_{1}-t\right)\left(\beta v_{h}+k v_{l}\right)\right)=\left(\gamma / 2\left(2+t_{1}\right)\right)\left((1+k / \beta) v_{l}+\left(t_{1}-t\right)\left(v_{h}+\right.\right.$ $\left.\left.k v_{l} / \beta\right)\right)$, it is clear that $q_{t}^{*}$ decreases with the increase of $\beta$. All the above can support Lemma 4 .

The result of Lemma 4 is illustrated in Figure 1 using numerical computations, where we plot the optimal ads volume with different values of $\beta$. As can be seen in Figure 1, the high ads-aversion coefficient corresponds to the low volume ads. For each curve of ads volume, the optimal number of ads is decreasing with the increase of time $t$. This result is rational in real online video market case. The longer the video available time lasts, the less attractive the video content is. Therefore, to get the sustainable profit in the long run, it is optimal for online providers to reduce ads volume.

Lemma 5. The number of viewers who select low-quality content $x_{l, t}$ increases as time $t$ increases, if $k v_{l}>\beta v_{h}$, when $t$ is in the time interval $\left(t_{0}, t_{1}\right)$.

Proof. In case II Stage 2, we obtain

$$
\begin{gathered}
x_{l, t}=\frac{\gamma v_{h}}{2\left(v_{h}-v_{l}\right)\left(2+t_{1}\right)}\left[\left(1-\frac{\beta}{k}\right)\left(t-t_{0}\right)\right. \\
\left.+\left(1-\frac{\beta v_{h}}{k v_{l}}\right)\left(t_{1}\left(t-t_{0}\right)-\frac{t^{2}-t_{0}^{2}}{2}\right)\right] .
\end{gathered}
$$

Take the first derivative of $x_{l, t}$ with respect to $t$, which leads to $\partial x_{l, t} / \partial t=\gamma v_{h} / 2\left(v_{h}-v_{l}\right)\left(2+t_{1}\right)\left[(1-\beta / k)+\left(1-\beta v_{h} / k v_{l}\right)\left(t_{1}-t\right)\right]$, given the condition $\beta / k<v_{l} / v_{h}$, and then obtain $\partial x_{l, t} / \partial t>0$, which supports Lemma 5.

Lemma 5 shows that, under the condition of $\beta / k<v_{l} / v_{h}$, if advertising price is high and the actual high-quality value is relatively low, viewers tend to prefer the free mode. Therefore, 


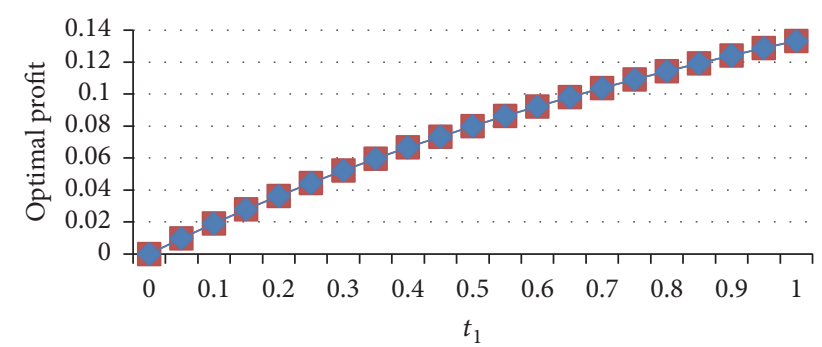

FIGURE 2: The optimal profit of case I with respect to time $t_{1}$.

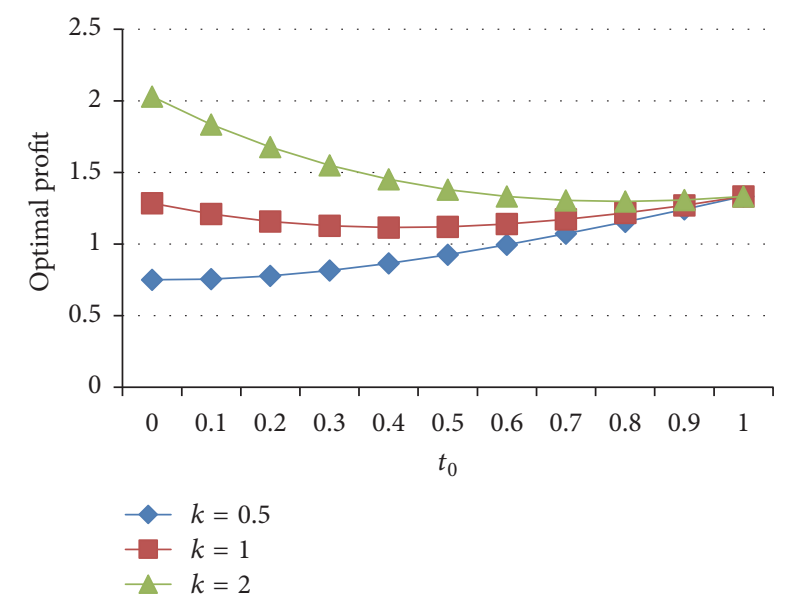

Figure 3: The optimal profit of case II with different ads prices $k$.

it is beneficial for online providers to adopt free strategy to generate advertising revenue. Taking this into account is significant for providers when fixing the optimal video price and ads volume.

To have a better understanding of parameters influence on $x_{l, t}$, we draw the curve of $x_{l, t}$ with respect to $\beta / k$ and $v_{h} / v_{l}$ in Figures 2 and 3. With generality, we set parameters values as $t_{0}=0.5, t_{1}=1$, and $\gamma=0.7$. Please see the explanations in the following section.

\section{The Optimal Profit}

According to the Hamiltonian principle and the previous analysis, we calculate the maximum profits taking into consideration the optimal strategies of fee charge and amount of ads. For case I fee mode, the optimal profit $\Pi_{\mathrm{I}}\left(t_{1}\right)$ equals

$$
\begin{aligned}
\Pi_{\mathrm{I}}\left(t_{1}\right) & =\int_{0}^{t_{1}} p_{t} \dot{x}_{h, t} d t=\int_{0}^{t_{1}} \frac{v_{h}\left(1+t_{1}-t\right)}{2+t_{1}} \cdot \frac{1}{2+t_{1}} d t \\
& =\frac{v_{h} t_{1}}{2\left(2+t_{1}\right)} .
\end{aligned}
$$

Figure 2 reflects the changing trend of the optimal profit for case I. We observe in this figure that the optimal profit increased as the time $t_{1}$ increased, but the increase is not very sharp. When $t_{1}$ tends to infinity, the maximum profit is obtained as $\Pi_{\mathrm{I}}=v_{h} / 2$. Also, we can observe from the equation of $\Pi_{\mathrm{I}}\left(t_{1}\right)$ that it is always optimal for online providers to adopt the single fee mode in time period $t_{1}$ when the high-quality value $v_{h}$ is increasing. For case II, the mixed fee-free mode, we calculate the total profit $\Pi_{\mathrm{II}}\left(t_{0}\right)$ as a function of the critical time $t_{0}$, separating into two stages. The respective profits are noted as $\Pi_{\mathrm{II}, 1}\left(t_{0}\right)$ and $\Pi_{\mathrm{II}, 2}\left(t_{0}\right)$ :

$$
\begin{aligned}
\Pi_{\mathrm{II}, 1}\left(t_{0}\right) & =\int_{0}^{t_{0}} p_{t} \dot{x}_{h, t} d t=\int_{0}^{t_{0}} \frac{v_{h}\left(1+t_{1}-t\right)}{2+t_{1}} \frac{1}{2+t_{1}} d t \\
& =\frac{v_{h}\left(2+2 t_{1}-t_{0}\right) t_{0}}{2\left(2+t_{1}\right)^{2}},
\end{aligned}
$$

and for Stage 2 profit, from the above obtained equations, we can have the following formulation:

$$
\begin{aligned}
& \Pi_{\mathrm{II}, 2}\left(t_{0}\right)=\int_{t_{0}}^{t_{1}}\left(\gamma p_{t}^{*} \dot{x}_{h, t}+k q_{t}^{*} \dot{x}_{l, t}\right) d t \\
& =\frac{\gamma^{2} v_{0}^{2}}{4\left(v_{0}-v_{1}\right)\left(2+t_{1}\right)^{2}}\left(( 1 + t _ { 1 } ) \left(-\frac{v_{1}}{v_{0}}-t_{1}\right.\right. \\
& \left.+\frac{\alpha v_{0}}{k v_{1}} t_{1}+\frac{\alpha v_{0}}{k v_{1}}\right)\left(t_{1}-t_{0}\right)+\left[\left(1-\frac{\alpha v_{0}}{k v_{1}}\right)\left(1+t_{1}\right)\right. \\
& \left.-\left(-\frac{v_{1}}{v_{0}}-t_{1}+\frac{\alpha v_{0}}{k v_{1}} t_{1}+\frac{\alpha v_{0}}{k v_{1}}\right)\right] \frac{t_{1}^{2}-t_{0}^{2}}{2}-(1 \\
& \left.\left.-\frac{\alpha v_{0}}{k v_{1}}\right) \frac{t_{1}^{3}-t_{0}^{3}}{3}\right)+\frac{\gamma v_{0}\left(1+t_{1}\right)}{2+t_{1}} C_{h}\left(e^{-t_{1}}-e^{-t_{0}}\right) \\
& -\frac{\gamma v_{0} C_{h}}{2+t_{1}}\left[e^{-t_{1}}\left(t_{1}+1\right)-e^{-t_{0}}\left(t_{0}+1\right)\right] \\
& +\frac{\gamma^{2} v_{0} v_{1} k}{4 \alpha\left(v_{0}-v_{1}\right)\left(2+t_{1}\right)^{2}}\left[\left(1-\frac{\alpha^{2}}{k^{2}}\right)\left(t_{1}-t_{0}\right)\right. \\
& +2\left(1-\frac{\alpha^{2} v_{0}}{k^{2} v_{1}}\right) t_{1}\left(t_{1}-t_{0}\right)-\left(1-\frac{\alpha^{2} v_{0}}{k^{2} v_{1}}\right)\left(t_{1}^{2}\right. \\
& \left.-t_{0}^{2}\right)+\left(1-\frac{\alpha^{2} v_{0}^{2}}{k^{2} v_{1}^{2}}\right) t_{1}^{2}\left(t_{1}-t_{0}\right)-\left(1-\frac{\alpha^{2} v_{0}^{2}}{k^{2} v_{1}^{2}}\right) \\
& \left.\cdot t_{1}\left(t_{1}^{2}-t_{0}^{2}\right)+\frac{1}{3}\left(1-\frac{\alpha^{2} v_{0}^{2}}{k^{2} v_{1}^{2}}\right)\left(t_{1}^{3}-t_{0}^{3}\right)\right] \text {. }
\end{aligned}
$$

Because the formulation is complicated, we investigate numerically the influence of critical time $t_{0}$ on the total profit $\Pi_{\mathrm{II}}\left(t_{0}\right)$ in case II. Without losing generality, set the total interval $t_{1}=1, \gamma=0.7, \beta=0.4, v_{l}=4$, and $v_{h}=8$, and $t_{0}$ belongs to $\left[0, t_{1}\right]$. The following shows numerically how the parameter of ads unit price $k$ influences the optimal profit of case II of the mixed fee-free mode in Figure 3.

It can be found that no matter how the unit ads income varies, the optimal profit strategy is to take into action only one business model, either the single fee mode or the mixed fee-free mode, as the profit reaches a maximum value at the ends of the interval. The optimal profit increases as $k$ increases. When the value of ads price $k$ is small, it suggests that the mixed fee-free mode profiting ads revenue is less 
efficient than the revenue of the single fee mode, which is supported in case $k=0.5$ in Figure 3. Clearly, when $t_{0}=0$, there exists the mixed fee-free mode; for $t_{0}=1$, there only exists the single fee mode. Please see the optimal profit curve with $k=0.5$; the profit at $t_{0}=0$ is less than that at $t_{0}=$ 1 , which means the single fee mode is optimal. Conversely, when the value of ads price $k$ is large, the mixed fee-free mode with ads revenue will create more profit. As shown in case $k=2$ in Figure $3, t_{0}=0$ reaches the maximum profit, which means the mixed fee-free mode outperforms in this case. At the same time, when $k=1$, the profits at both ends of the interval are nearly equal, while the profit decreases as $t_{0}$ varying in the interval. This means, for online providers, there is no difference between the single fee mode and mixed feefree mode. Therefore, the optimal strategy is to adopt either of them. Finally, from the above analysis, it can be concluded that the online providers should set their strategies according to the unit ads price $k$, but the strategy should always be one single business model.

\section{Conclusion}

This paper has investigated the optimal strategy for online video service business. Considering the dynamic decisions in real video market, we divide the whole period into the single fee phase and mixed fee-free phase and then, respectively, create continuous time model for each. Our models have presented a straightforward way to study the provider's pricing strategy and advertising decision based on viewers' emotional factor. There is a significant influence among applied price, ads volume, and video content quantity. In the single fee mode, we have obtained the optimal time-varying price which maximizes the profit from video service within a certain time interval. The result implies that the optimal price of online video increases with high-quality value while decreasing with time. In the mixed fee-free mode, we have found out the optimal fee and ads volume. It is beneficial for online providers to choose higher charge fee and advertising intensity. Besides, the price and the number of ads decrease as ads-aversion coefficient increases. It has turned out that, in a long run period of time, taking one strategy either fee mode or free mode is optimal for the online providers.

For future research, the following subjects can be carried out in three aspects:

(i) Accomplishment of the model: There are other factors which determine the revenue model. For example, in Ren et al. [21], the authors point out that the online popularity is a significant effecting factor on videos in online systems; in Iveroth et al. [22], competitors' price is a base factor for deciding; in Choi et al. [23], they imply that discount on price can have effect on the sales of information goods; in another study, Niu and $\mathrm{Li}$ [24] suggest for the Internet service providers a price model depending on the congestion of Internet.

(ii) Model validation: our time-continuous model should fit the data collected in real world, identify model coefficients, and validate other sets of data. As mentioned in Walz et al. [25], the uncertainty analysis could be carried out in two aspects: the model form uncertainty and the parameters uncertainty. The parameters standard error is investigated and the values should fall into the confidence interval. In this way, the optimal time-varying price solutions are validated.

(iii) Another path to investigate online video services is predicting and analyzing based on a fitted nonparametric model. There are several advanced regression methods to be investigated, such as multivariate adaptive regression splines (MARS) or its alternative CMARS proposed by Weber et al. [26] or RCMARS proposed by Özmen et al. [27]. The prediction model can be used for simulation and cross validation with respect to our models.

\section{Conflicts of Interest}

The authors declare that they have no conflicts of interest.

\section{References}

[1] R. K. Chellappa and S. Shivendu, "Managing piracy: Pricing and sampling strategies for digital experience goods in vertically segmented markets," Information Systems Research, vol. 16, no. 4, pp. 400-417, 2005.

[2] S. Kumar and S. P. Sethi, "Dynamic pricing and advertising for web content providers," European Journal of Operational Research, vol. 197, no. 3, pp. 924-944, 2009.

[3] T. R. Weiss, "Fees for online content proliferating, but will users buy," Computer World, 2002.

[4] C. Shapiro and H. R. Varian, Information rules: A strategy guide to the network economy, Harvard Business School Press, Boston, MA, 1998.

[5] H. K. Cheng and Q. C. Tang, "Free trial or no free trial: Optimal software product design with network effects," European Journal of Operational Research, vol. 205, no. 2, pp. 437-447, 2010.

[6] D. Halbheer, F. Stahl, O. Koenigsberg, and D. R. Lehmann, "Choosing a digital content strategy: How much should be free?" International Journal of Research in Marketing, vol. 31, no. 2, pp. 192-206, 2014.

[7] F. J. Riggins, "Market segmentation and information development costs in a two-tiered fee-based and sponsorship-based web site," Journal of Management Information Systems, vol. 19, no. 3, pp. 69-86, 2002.

[8] A. Prasad, V. Mahajan, and B. Bronnenberg, "Advertising versus pay-per-view in electronic media," International Journal of Research in Marketing, vol. 20, no. 1, pp. 13-30, 2003.

[9] D. Halbheer, F. Stahl, O. Koenigsberg, and D. R. Lehmann, “Digital content strategies," Marketing Letters, vol. 25, no. 3, pp. 331-341, 2013.

[10] M. Peitz and T. M. Valletti, "Content and advertising in the media: Pay-tv versus free-to-air," International Journal of Industrial Organization, vol. 26, no. 4, pp. 949-965, 2008.

[11] C. Kwon, "Single-period balancing of pay-per-click and payper-view online display advertisements," Journal of Revenue and Pricing Management, vol. 10, no. 3, pp. 261-270, 2011.

[12] M. Lin, X. Ke, and A. Whinston, "Vertical differentiation and a comparison of online advertising models," Journal of 
Management Information Systems, vol. 29, no. 1, pp. 195-236, 2012.

[13] R. M. Dewan, M. L. Freimer, and J. Zhang, "Management and valuation of advertisement-supported web sites," Journal of Management Information Systems, vol. 19, no. 3, pp. 87-98, 2002.

[14] H. S. Na, J. Hwang, J. Y. J. Hong, and D. Lee, "Efficiency comparison of digital content providers with different pricing strategies," Telematics and Informatics, vol. 34, no. 2, pp. 657663, 2017.

[15] H. L. Vogel, Entertainment Industry Economics, Cambridge Books, New York, NY, USA, 2015.

[16] R. Casadesus-Masanell and F. Zhu, "Strategies to fight adsponsored rivals," Management Science, vol. 56, no. 9, pp. 14841499, 2010.

[17] K. C. Wilbur, "A two-sided, empirical model of television advertising and viewing markets," Marketing Science, vol. 27, no. 3, pp. 356-378, 2008.

[18] S. P. Anderson and S. Coate, "Market provision of broadcasting: a welfare analysis," Review of Economic Studies, vol. 72, no. 4, pp. 947-972, 2005.

[19] D. W. Yeung and L. A. Petrosyan, Cooperative Stochastic Differential Games, Springer Series in Operations Research and Financial Engineering, Springer, New York, NY, USA, 2006.

[20] S. P. Sethi and G. L. Thompson, Optimal Control Theory: Applications to Management Science and Economics, Springer Science \& Business Media, 2006.

[21] Z.-M. Ren, Y.-Q. Shi, and H. Liao, "Characterizing popularity dynamics of online videos," Physica A: Statistical Mechanics and its Applications, vol. 453, pp. 236-241, 2016.

[22] E. Iveroth, A. Westelius, C.-J. Petri, N.-G. Olve, M. Cöster, and F. Nilsson, "How to differentiate by price: Proposal for a fivedimensional model," European Management Journal, vol. 31, no. 2, pp. 109-123, 2013.

[23] H. Choi, D. Medlin, and S. Hunsinger, "The effects of discount pricing strategy on sales of software-as-a-service (SaaS): online video game market context," Journal of Information Systems Applied Research, vol. 10, no. 1, pp. 55-64, 2017.

[24] D. Niu and B. Li, "Congestion-aware internet pricing for media streaming," in Proceedings of the 2014 IEEE Conference on Computer Communications Workshops, INFOCOM WKSHPS 2014, pp. 571-576, Toronto, ON, Canada, May 2014.

[25] N. Walz, M. Burkhardt, P. Eberhard, and M. Hanss, "A Comprehensive Fuzzy Uncertainty Analysis of a Controlled Nonlinear System With Unstable Internal Dynamics," ASCEASME Journal of Risk and Uncertainty in Engineering Systems, Part B: Mechanical Engineering, vol. 1, no. 4, Article ID 041008, pp. 1-12, 2015.

[26] G. W. Weber, I. Batmaz, G. Köksal, P. Taylan, and F. YerlikayaÖzkurt, "CMARS: A new contribution to nonparametric regression with multivariate adaptive regression splines supported by continuous optimization," Inverse Problems in Science and Engineering, vol. 20, no. 3, pp. 371-400, 2012.

[27] A. Özmen, G. W. Weber, I. Batmaz, and E. Kropat, "RCMARS: Robustification of CMARS with different scenarios under polyhedral uncertainty set," Communications in Nonlinear Science and Numerical Simulation, vol. 16, no. 12, pp. 4780-4787, 2011. 


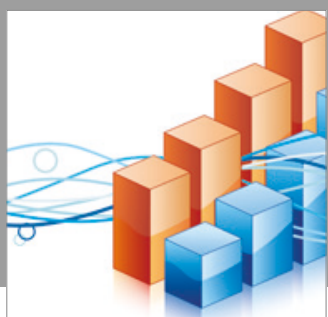

Advances in

Operations Research

vatersals



\section{The Scientific} World Journal
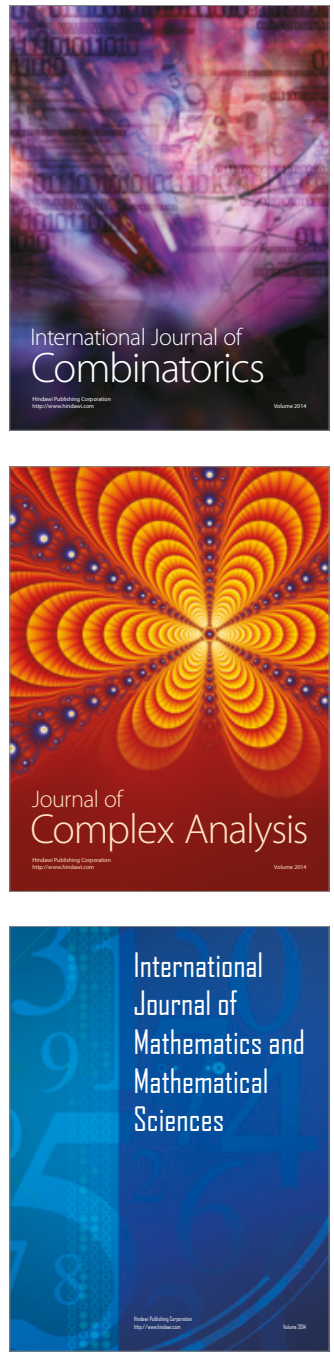
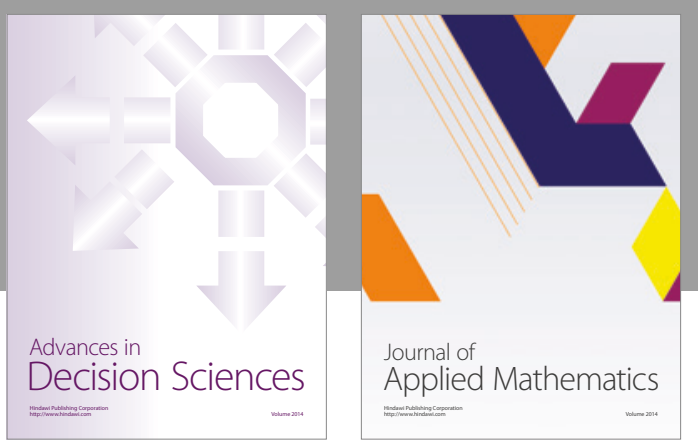

Algebra

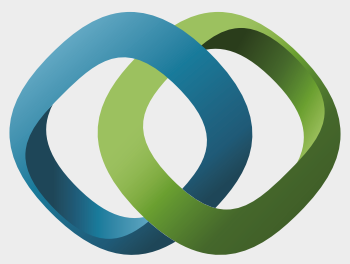

\section{Hindawi}

Submit your manuscripts at

https://www.hindawi.com
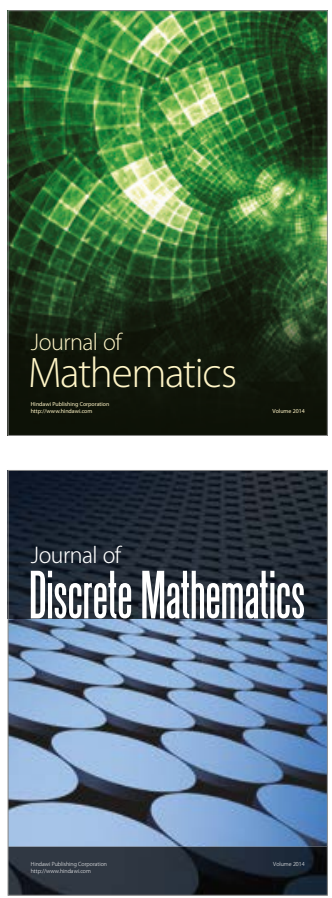

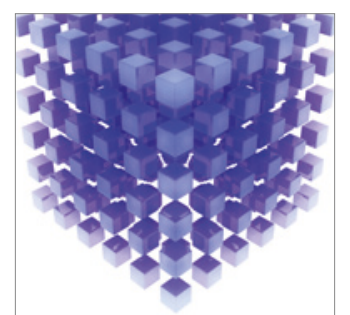

Mathematical Problems in Engineering


Journal of

Function Spaces

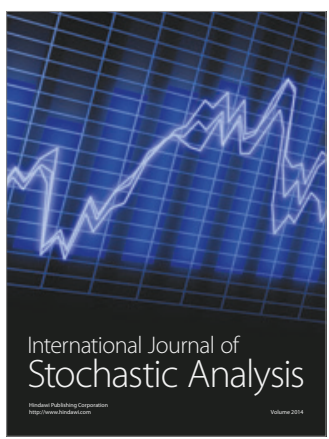

Probability and Statistics
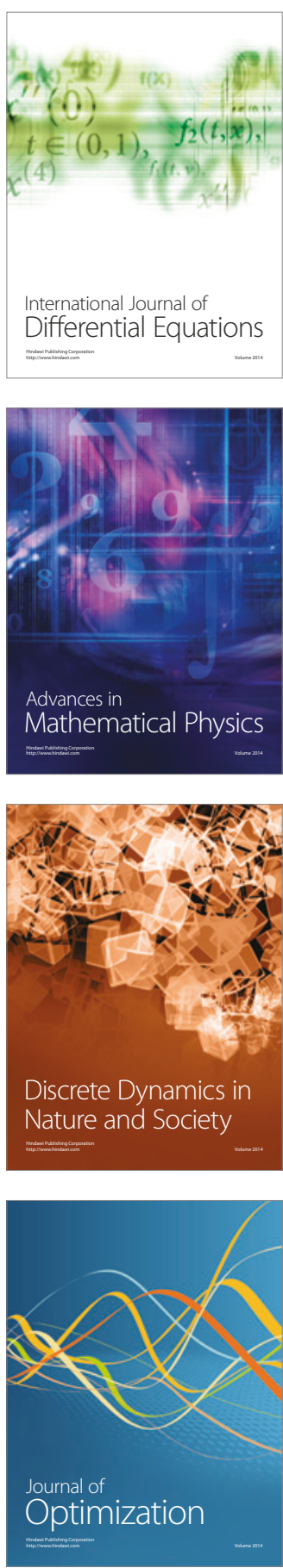